\title{
Pulsatile Flow in the Choroidal Circulation: A Preliminary Inyestigation
}

\author{
R. W. FLOWER ${ }^{1}$ and G. J. KLEIN ${ }^{2}$ \\ Maryland, USA
}

\begin{abstract}
Summary
A preliminary investigation has been made of choroidal blood flow using a computeraided image analysis approach to interpretation of indocyanine green (ICG) dye choroidal angiograms. The goal of the study was to characterise blood flow through the choroidal arteries $v s$. choroidal capillaries and veins. The methods of analysis used are briefly reviewed, and preliminary data obtained mainly from monkey eyes are presented. Preliminary conclusions are made regarding the relationship between compliance of choroidal arterial vessels and blood flow through them.
\end{abstract}

Unlike sodium fluorescein angiography in which visualisation of the choroidal vasculature is hampered by presence of macular xanthophyll, retinal pigment epithelium and choroidal pigment, indocyanine green (ICG) dye angiograms are made using near-infrared wavelengths which penetrate these optical barriers; details of the ICG technique have been published previously. ${ }^{1,2}$ Data for this study of choroidal blood flow were derived from ICG angiograms made of $50^{\circ}$ fundus fields at a rate of 15 frames per second. Because usually only one angiographic study is available from each human subject, data were derived mainly from primate eyes of which multiple angiograms could be made under controlled conditions.

Due to the thick multi-layered structure of the choroidal vasculature and the greater blood velocity through it (compared to retinal blood flow velocity), interpretation of chor- oidal angiograms does not readily lend itself to the same by-eye-only evaluation used to interpret fluorescein angiograms. To aid in analysis of ICG angiograms, a relatively inexpensive personal computer-based image analysis system (comprised of only commercially available hardware components) and appropriate data analysis algorithms and software were developed. This approach to angiogram analysis is, to the best of our knowledge, the first to provide routine characterisation of the choroidal circulation in a way that conserves spatial distribution of blood flow dynamics across the entire choroidal area observed. Descriptions of the hard- and soft-ware used are detailed elsewhere, ${ }^{3}$ but the following brief overview of the data analysis approach used in this study is appropriate.

It is appropriate also to emphasise that the results presented in this paper should be considered preliminary.

*This investigation was supported in part by the US Navy under Contract N00039-87-C-5301 and by Becton Dickinson Microbiological Systems, Cockeysville, MD.

From: The Applied Physics Laboratory ${ }^{1,2}$ and the Wilmer Ophthalmological Institute ${ }^{1}$ of The Johns Hopkins University and Hospital.

Correspondence to: R W Flower, The Johns Hopkins University Applied Physics Laboratory, Johns Hopkins Road, Laurel, Maryland 20707, USA. 


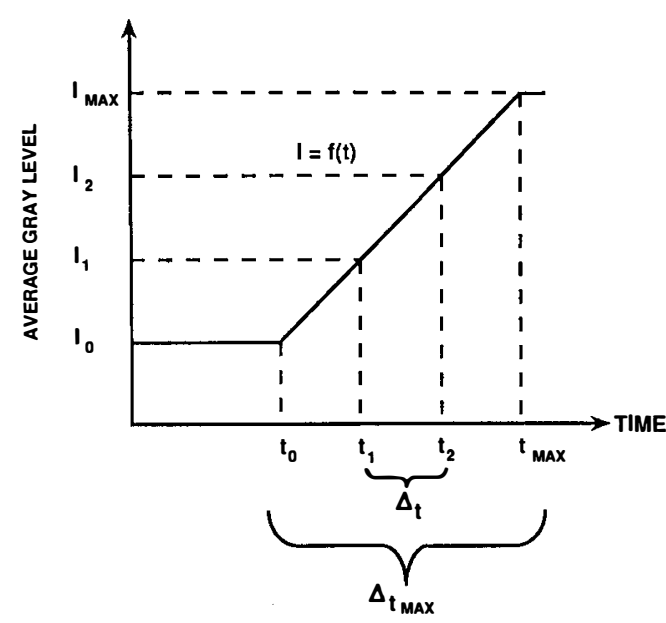

Fig. 1.

\section{Time Sequence Analysis of Digitized Angiogram Images}

Each high resolution video angiogram frame is recorded real-time as a 480 by 512 pixel by 256 gray level array (one pixel in this array corresponds to approximately a 20 micron spot on the fundus). Successive frames of the digitised angiographic sequence are precisely aligned with each other by software, and each frame is then divided into a grid pattern of up to $50 \times 50$ segments. For this preliminary study, however, a more course division into $26 \times 32$ segments was sufficient (one such segment corresponds approximately to the 0.25 $\mathrm{mm}^{2}$ area of a choriocapillaris lobule). The average grey level of all the pixels in each grid segment was computed for each segment in each angiogram frame, and this formed the data base subsequently analysed.

\section{Data Analysis and Display Algorithms}

Analysis of recorded average gray level data is based upon the following model: Each segment into which the choraid is divided defines a separate elastic compartment having one or more inlets (arterioles) and one or more outlets (veinules). The volume of a compartment is considered to be the aggregate volume of all the vessels and vessel segments contained in a full-thickness plug of choroidal tissue, bounded by the projections of the four lines which define the sides of the sector; compartment volume does not include intervascular spaces.
At any given time, each choroidal compartment underlying each segment is associated with a mean dye concentration and hence with a total level of dye fluorescence as well. Assuming that maximum dye concentration remains below that at which fluorescence quenching occurs, ${ }^{4}$ during the dye-filling phase the total fluorescence from any particular choroidal segment will be a monotonically increasing function of the total amount of dye within the compartment underlying that segment. That is, the fluorescence from a given segment results from the equal superposition of all vessels, regardless of depth from the retina. It is additionally assumed that the ICG dye is uniformly mixed with blood by the time it reaches the eye, that the wavefront of dye-tagged blood entering the ocular vasculatures is well defined, that the bolus of dye-tagged blood remains integral during its passage through the choroidal vasculature, and that the volume of the dyetagged blood bolus is greater than that of the volume of choroidal vasculature being observed.

Figure 1 shows an idealised characterisation of the average time-varying grey level for a single sector of a choroid. This plot indicates that the average grey level remains at some background level, $\mathrm{I}_{0}$, until the first appearance of dye occurs at time $t_{0}$. Once dye enters the compartment under this sector, average grey level increases to a maximum level, $\mathrm{I}_{\max }$, at time $t_{\max }$, when the compartment is completely filled with dye. $I_{1}, I_{2}, t_{1}$ and $t_{2}$ indicate arbitrary intermediate grey levels and the times at which they occur. Since the average grey level of a sector is a time-varying function, $I=f(t)$, the instantaneous filling rate of a sector is the first derivative of that function,

$$
\frac{\mathrm{dI}}{\mathrm{dt}}=\frac{\mathrm{df}(\mathrm{t})}{\mathrm{dt}} \text {. }
$$

In terms of the model, changes in this rate are induced by blood pressure gradients and by changes in the dye concentration.

Figure 2 schematically demonstrates derivation of the curve shown in Figure 1. The upto-32 fundus images from a typical angiographic sequence are shown stacked one upon the other and divided into grids, and the volume of choroidal vessels underlying a grid segment is shown to the left of the first angio- 


\section{Time Sequence Analysis}

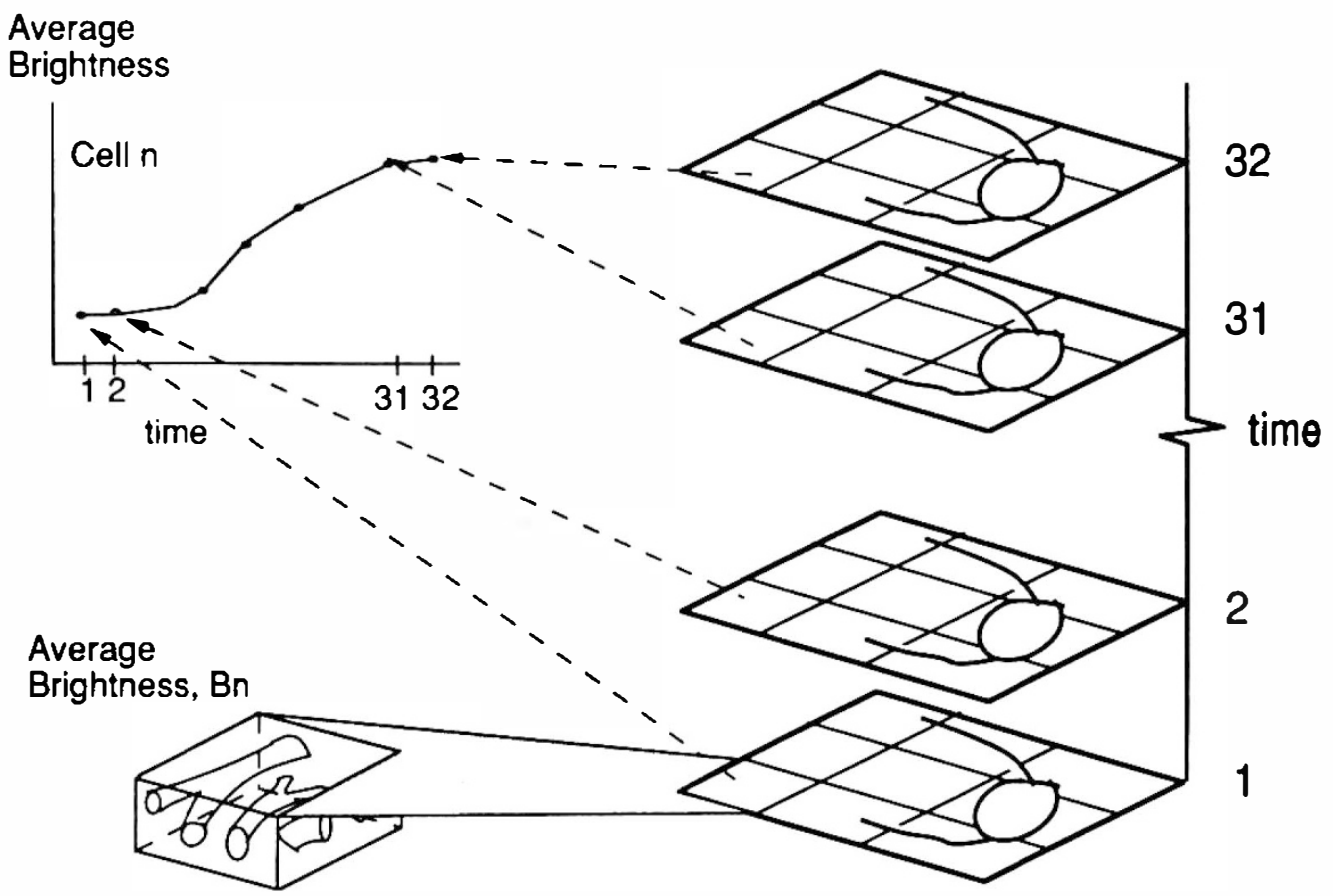

Fig. 2.

gram frame. The curve in the upper left-hand corner of the figure indicates the time-varying average brightness of one representative segment throughout the sequence of angiograms; such curves are generated for each sector in the grid. From these curves, various blood flow parameters, like instantaneous filling rate, can be derived.

In order to facilitate comparisons of various parameters computed for each of the sectors comprising the entire observed fundus area, it is convenient to visualise those parameters in terms of three-dimensional surfaces projected above the fundus area. Any blood flow parameter can be represented for each sector as a vector normal to the plane of the choroid, having its origin at the sector's centre. Pointto-point connection of each vector end to all neighbouring vector ends approximates a 3-dimensional surface representing that particular choroidal blood flow parameter for the entire fundus area observed; this process is shown schematically in Figure 3. For example, if the parameter of interest were instantaneous dye filling rate, then the elevated portions of the 3-dimensional surface would correspond to choroidal areas having rapid dye filling and lower portions to areas of less rapid filling. Once defined, a 3-dimensional surface can easily be rotated in space about any axis for inspection or to facilitate comparison to other such surfaces. Also, the amplitude of the vectors defining the surface can be changed freely to facilitate these comparisons since it is only their relative magnitudes with respect to each other which are of interest.

Since average grey level of a given sector is associated with the amount of dye in that sector at a given time, the first time derivative of the time-varying average grey level produces a curve representing the filling rate of those sectors. This is demonstrated in Figure 4 for a $3 \times 3$ array of sectors, throughout a sequence of angiogram images. The important thing to note is the cyclical variation in the curves which is of precisely the same frequency as the subject's heart rate. It was found that the 


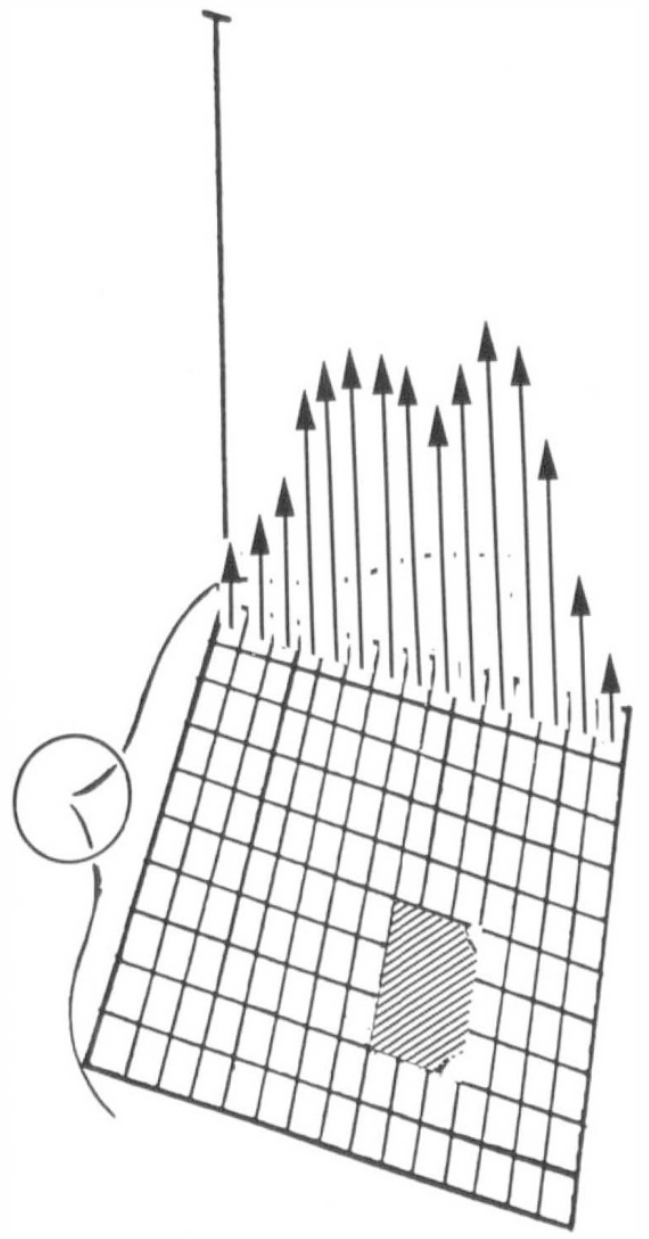

Fig. 3.

same three-dimensional surface representing instantaneous choroidal filling rates is generated from angiographic data recorded during each time interval corresponding to a systole. Moreover, the topography of these surfaces appear to be quite stable from day to day for a given individual, indicating that a threedimensional representation of average systolic filling rate may uniquely characterise the particular choroidal circulation from which it was derived. ${ }^{3}$

\section{Pulsatile Flow Through the Choroid}

For purposes of discussing a specific choroidal blood flow parameter, the more precise differentiation between individual grid segments provided by a 2 -dimensional overhead view is

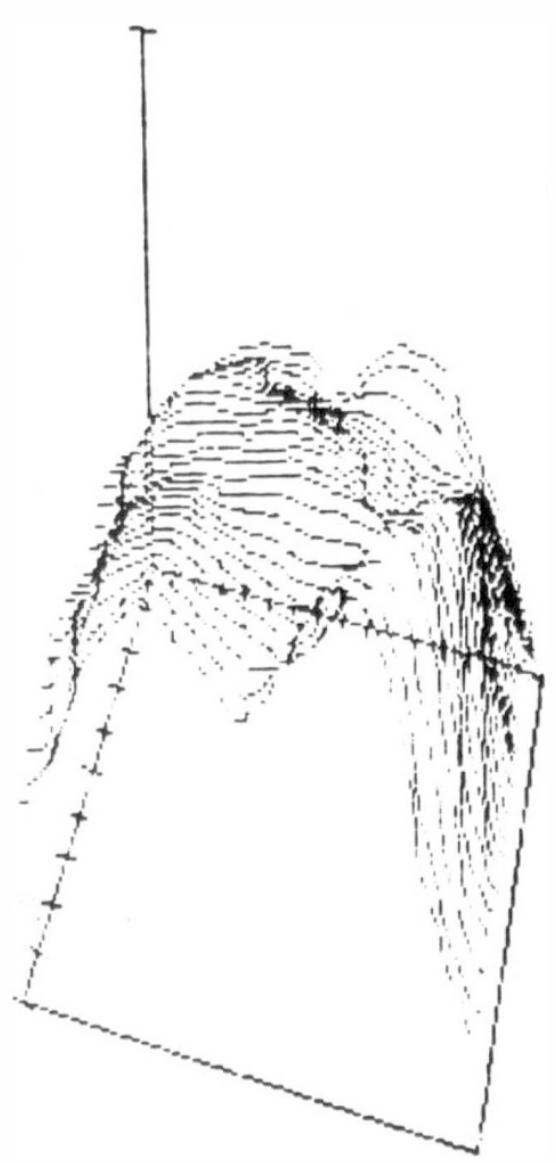

useful. Three successive instantaneous choroidal dye filling rate surfaces are shown in Figure 5 along with overhead views of the fundus area beneath each surface. The dots in these latter diagrams represent the centres of the grid segments into which the angiographic images were divided; the vectors emanating from some of those dots indicate approximately the relative velocities of the threedimensional surface at each instance. Diagrams like these made of a number of subjects, indicate that dye filling of the choroid occurs in a pulsatile fashion with dye entering the choroidal vasculature at points corresponding to the insertions of ciliary arteries (the x's) and then flowing radially away from those points. 

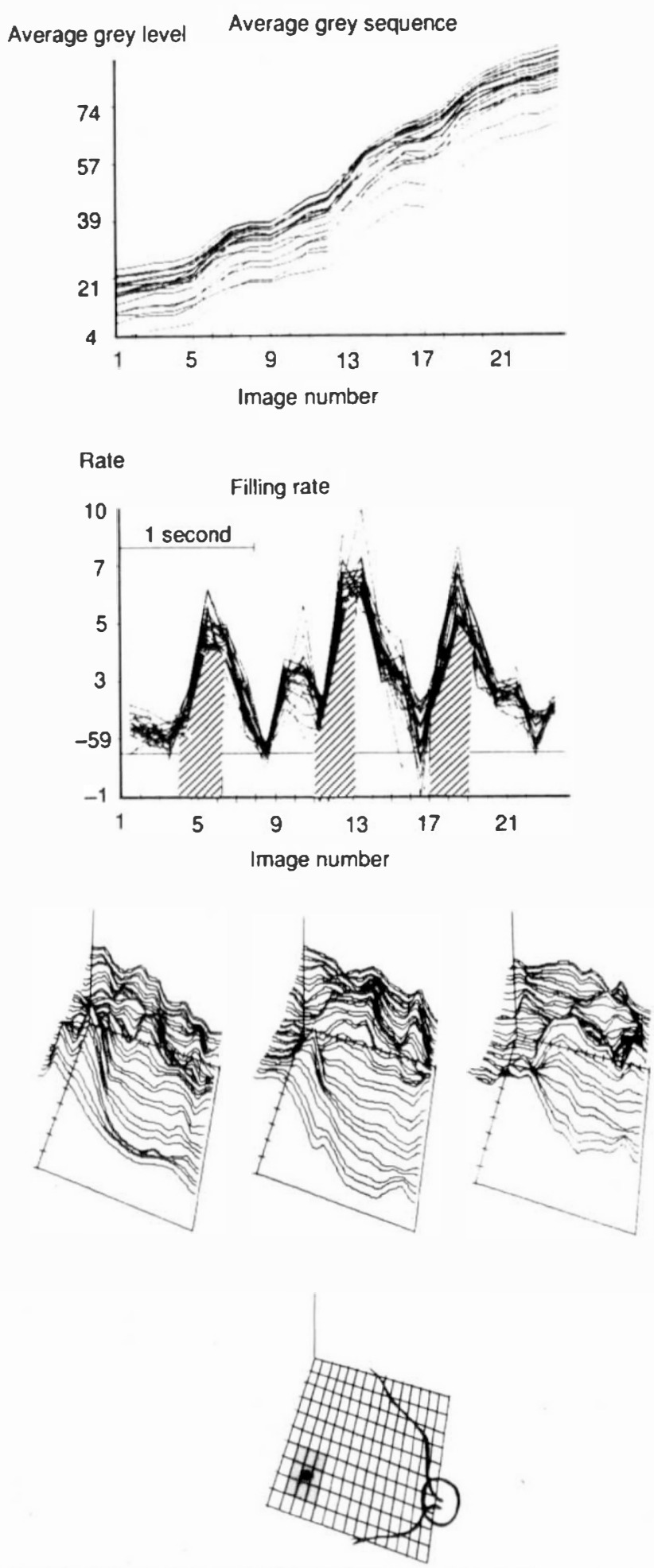

Fig. 4. 

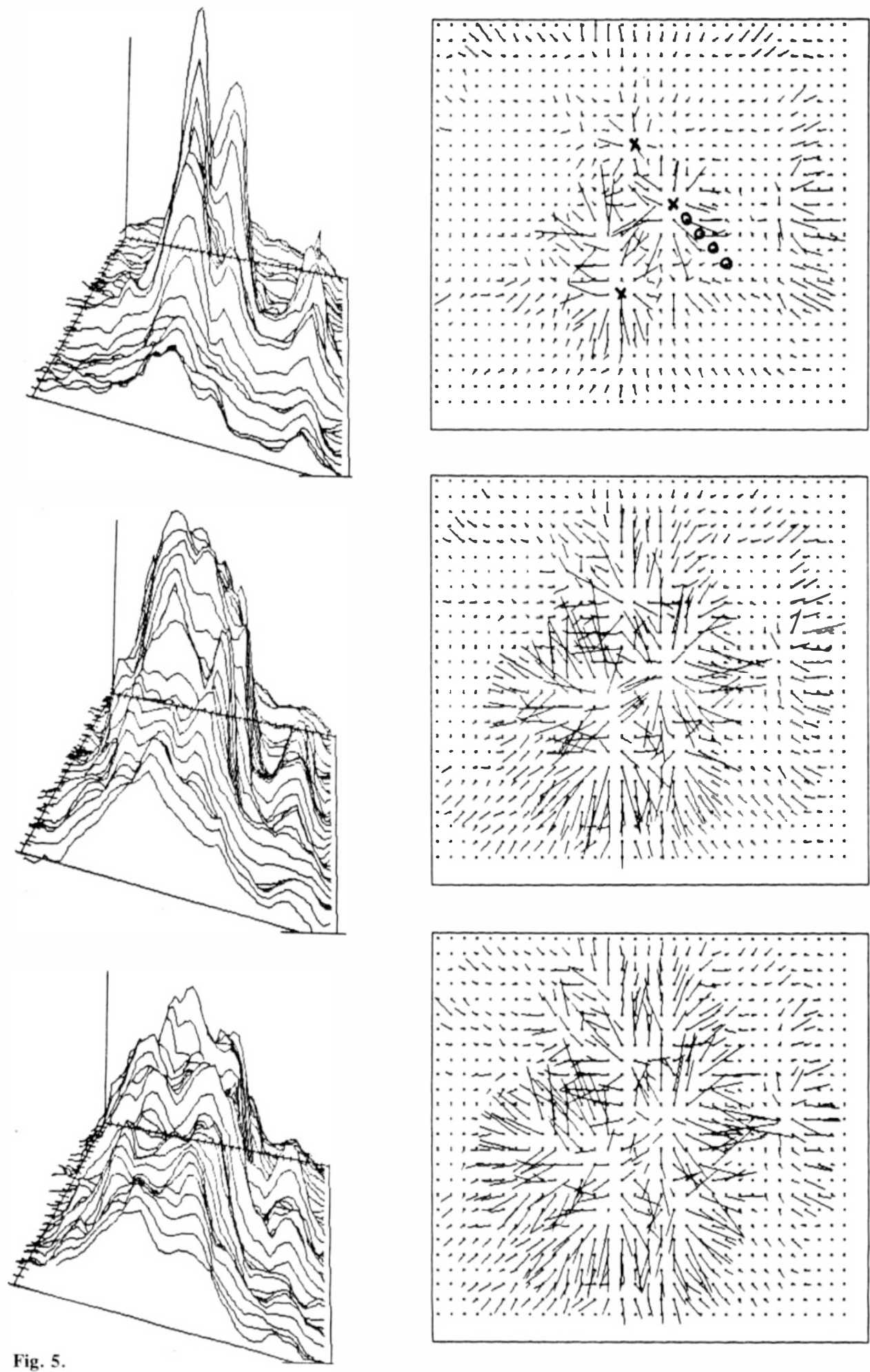


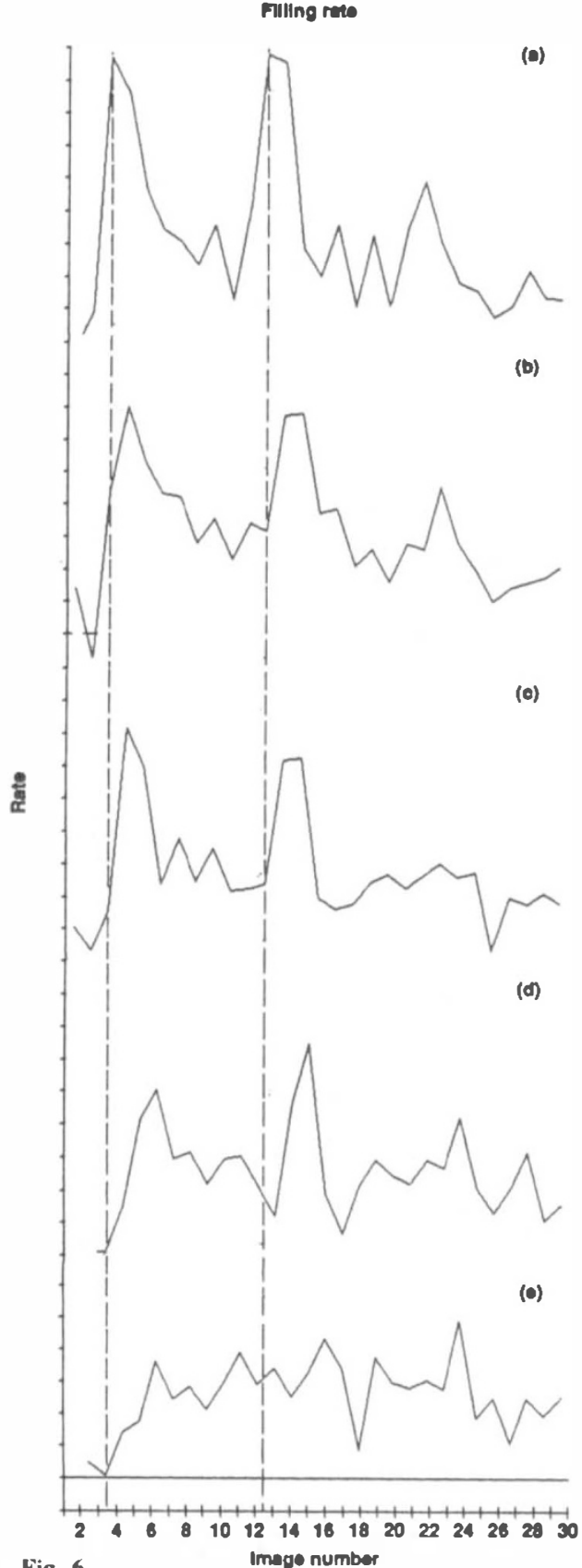

Fig. 6.

Time-varying rates of dye flow through different segments of the choroidal vasculature during the same time interval are shown in Figure 6 . The curves correspond to locations on the fundus (marked by circles) displaced radially at increasingly greater distances from the first location (marked by an $\mathrm{X}$ ) which overlies a short posterion ciliary artery insertion, curve (a). The waveform of curve (a) very closely resembles that measured by others in the carotid artery, ${ }^{5}$ in that each pulse contains a dicrotic notch. Such notches are not ordinarily found within the cardiovascular tree at points more proximal to the heart than the carotid artery, indicating that compliance of the arterial vessels between the carotid artery and the choroid are small enough that apparently little distortion of the blood flow waveform occurs in them.

It should also be noted that variations in amplitudes of successive pulses result from variation in the dye concentration in blood as the dye bolus moves through the choroid. Not surprisingly, as distance from the ciliary artery insertion point increases, a phase shift occurs in the flow pulses as a result of increased flow resistance. At the same time, the waveform is significantly distorted, indicating a difference in compliance between choroidal arterial vessels and the capillaries and veins.

A fairly easy way to change vascular compliance is to vary arterial $\mathrm{pO}_{2}$ and $\mathrm{pCO}_{2}$ levels by breathing different humidified gas mixtures prior to angiography. This was done in several anesthetised rhesus monkeys; typical results are shown for one monkey in Figure 7. Differences in the 3-dimensional surfaces representing instantaneous blood flow rates during systole indicate the significant changes induced by $\mathrm{O}_{2^{-}}$and $\mathrm{CO}_{2}$-breathing. Compared to the air-breathing case, the net effect of oxygen breathing is generally reduced blood flow, while that of $\mathrm{CO}_{2}$-breathing is generally increased blood flow. Of greater interest, however, is the change in relationship between blood flow waveforms in the choroidal arteries and the choriocapillaryl venous bed. This is demonstrated by the two sets of blood flow curves corresponding to each gas-breathing state. On each 3-dimensional surface in Figure 7 are two darkened squares identifying the same two areas on the subject choroid; the area under each square comprises a $3 \times 3$ array of grid segments from which the nine curves in each set were produced.

The sets of curves for the air-breathing state bear the same relationship demonstrated in 

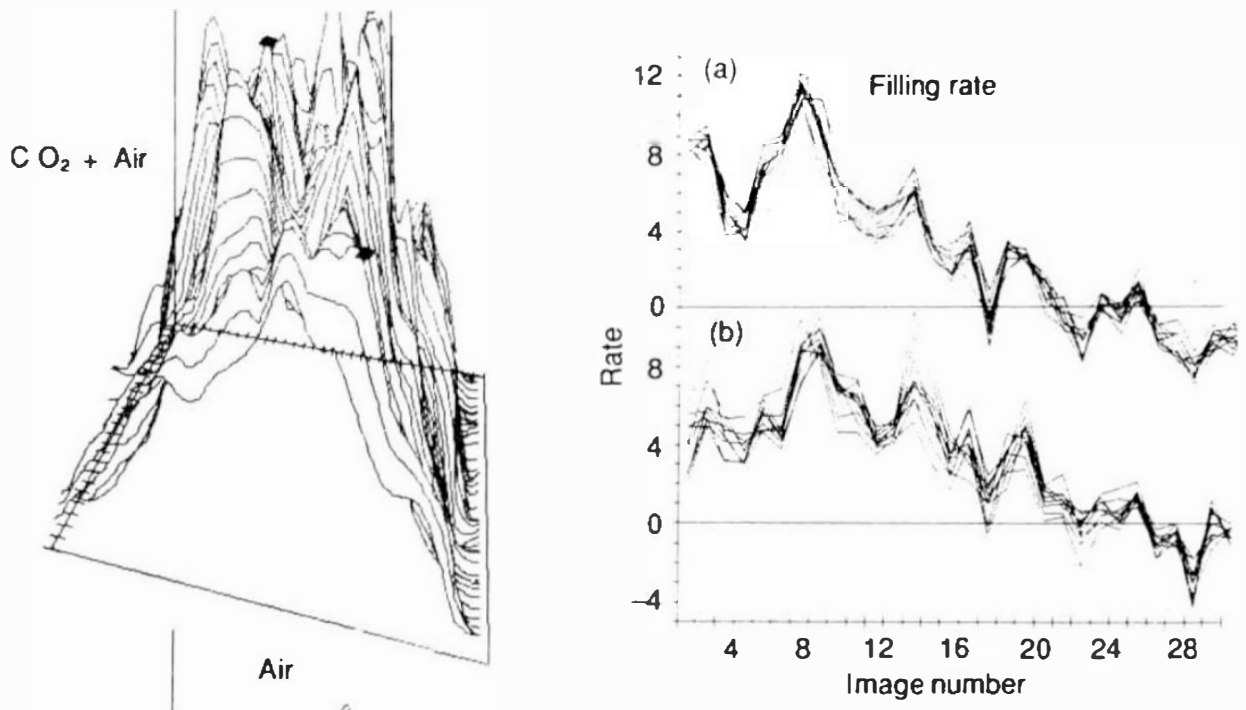

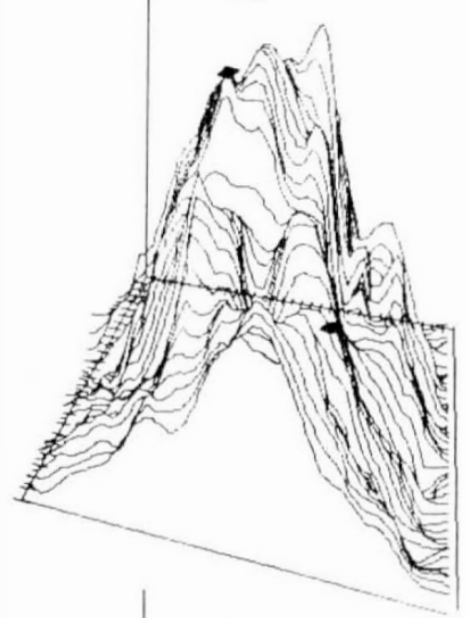

$\mathrm{O}_{2}$

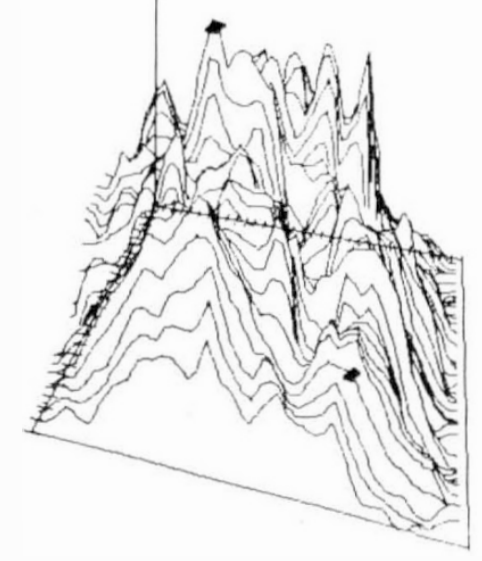

Fig. 7 .

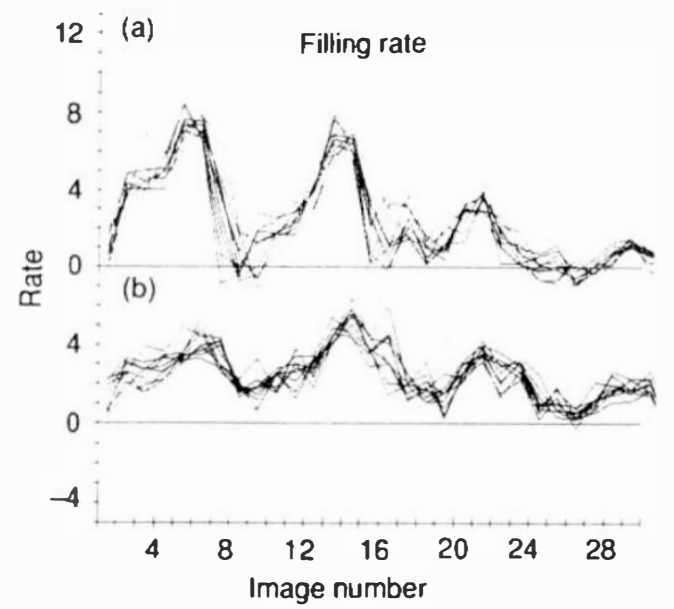

12: (a)

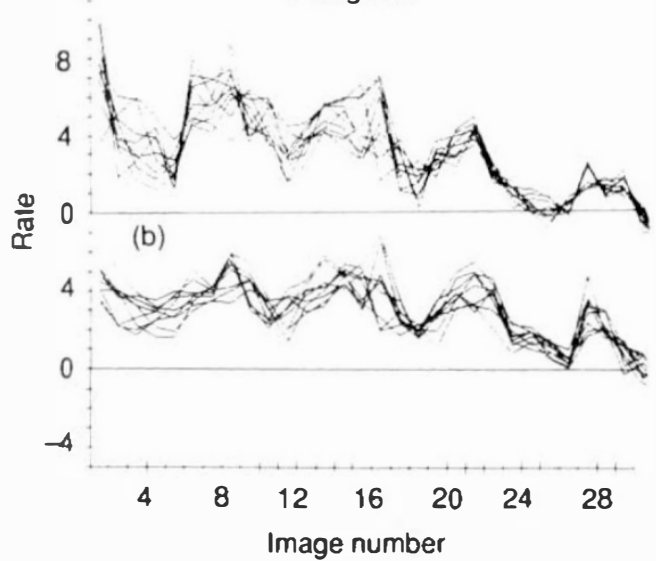


Figure 6; namely, compared to the waveform of blood flow above a short posterior ciliary artery (curve a), the waveform of blood flow at a distal location (curve b) is of reduced amplitude, phase-shifted, and slightly distorted, as might be expected for vascular beds having different compliances. In the $\mathrm{CO}_{2}$ breathing case, the pulse amplitudes at the ciliary artery and distal location are nearly identical, and the pulses are nearly in phase; but, the waveform is different from that of the air-breathing case in that the rise-and-decay times of the $\mathrm{CO}_{2}$ pulses are shorter. This might be explained by $\mathrm{CO}_{2}$-breathing having increased the compliance normally present in arterial vessels by causing them to dilate. This could result in greater blood flow through the choriocapillary/venous bed due to increased capacity of all the arterial vessels influencing that bed. This also could result in similar flow waveforms at the two locations, both of which more resemble the arterial vessel waveform of the air-breathing case than the capillary/ venous bed waveform.

Oxygen-breathing, on the other hand, reduces arterial vessel wall compliance by inducing vasoconstriction. The resulting higher resistance to blood flow would be expected to reduce the ciliary artery flow pulse amplitude (compared to the air-breathing case), and apparently it does so. Again, the arterial blood flow waveform and that of the capillary/venous bed are similar and exhibit no phase shift. In this case, however, the two sets of curves more resemble the capillary/venous bed waveform of the air-breathing case than the arterial waveform.

\section{Conclusions}

Results from this preliminary study demon- strate the feasibility of using methods which may be applied to human subjects as. well as monkeys to investigate choroidal blood flow. Conclusions regarding parameters, such as compliance, related to specific types of choroidal vessels is possible, as demonstrated. Although the preliminary results presented here may be considered somewhat intuitive, we believe this study has produced the first in vivo demonstration of the relationships between compliances of choroidal arterial vessels and the rest of the choroidal vascular bed. That those relationships apparently can be influenced by other physiological parameters such as blood gas content has also been demonstrated. This suggests that the influence of other parameters, including blood pressure, blood viscosity and intraocular pressure, may also be investigated using this same approach.

\section{References}

${ }^{1}$ Flower RW and Hochheimer BF: A clinical technique and apparatus for simultancous angiography of the scparate retinal and choroidal circulations. Invest Ophthalmol 1973 12: 248-61.

${ }^{2}$. Bischoff $P$ and Flower RW: Ten years' expcrience with choroidal angiography using indocyanine green dye: A new routine examination or an cpiloguc? Doc Ophthalmol 1985 60: 235-91.

${ }^{3}$. Klien GJ, Baumgartner RH, Flower RW: Characterization and classification of choroidal blood flow. Invest Ophthalmol Vis Sci (in press).

${ }^{+}$Flower RW and Hochheimer BF: Quantification of indicator dye concentration in ocular blood vessels. Exp Eye Res 1977 25: 103-111.

${ }^{5}$ Spencer MP and Denison AB: Pulsatile blood flow in the vascular system. In: Hamilton WF and Dow $P$ (eds.) Handbook of physiology. Circulation section, Baltimore, 1962, The Williams and Wilkins Co., Vol.1. 